\title{
Memorizing 2D Tactile Right-Angle-Shapes by Congenitally Blind and Sighted Adults
}

\author{
Magdalena Szubielska, Emilia Zabielska-Mendyk \\ The John Paul II Catholic University of Lublin \\ Lublin, Poland
}

\begin{abstract}
The study was designed to compare the ability of congenitally blind and sighted individuals to memorize embossed 2D right-angle-figures with varied number of angles displayed against a grid or in a frame. We hypothesized that blind adults learn embossed shapes: 1) faster than sighted participants - this assumption was verified positively; 2) more accurately - which was not confirmed. The grid interfered with sighted people in solving the task, but it had no impact on the performance of participants with blindness. These results can be explained by referring to the memorizing strategies used by those who do and do not have visual experience. Sighted individuals use visual strategies more often than congenitally blind participants. The strategies identified in both groups were used either in isolation or in combination with a verbal or a kinesthetic strategy.
\end{abstract}

Key words: visuo-spatial processes, touch, imagery strategies, blindness

The purpose of this study was to examine the ability of congenitally blind and sighted individuals to memorize spatial information presented in the form of raised-line images of geometric patterns - right-angle-shapes, with varying numbers of angles presented against a grid or in a frame. We were particularly interested in the amount of time needed for learning the tactile figures, the shapes recognition accuracy, and the mental imagery strategies used by both groups.

Acknowledgments

This work was supported by Ministry of Science and Higher Education, Poland, grant no. N N106 064235. We want to thank Marcin Machowski for his help in data collection and Professor Bogusław Marek and his team for their help in preparing the tactile stimuli.

Correspondence concerning this article should be addressed to Magdalena Szubielska, Ph.D., The John Paul II Catholic University of Lublin, Institute of Psychology, Aleje Raclawickie 14, Lublin, 20-950, Poland. E-mail: magdasz@kul.pl

Received August 3, 2017
Previous research findings show differences between blind and sighted individuals in the processing of two-dimensional shapes explored by touch, interestingly often suggesting blind participants' advantage in this matter. Blind participants are more successful than sighted participants in differentiating two-dimensional angles (Alary et al., 2008), identifying curvatures (Davidson, 1972), naming basic embossed geometrical figures (i.e., square, rectangle, triangle) (Theurel et al., 2012), distinguishing a figure from the background and naming incomplete raised-line shapes (Ballesteros et al., 2005). Furthermore, in comparison to sighted individuals, blind participants are faster to match shapes of geometrical figures to their cut-outs in a board (Postma et al., 2007) and to distinguish figures embedded against a background in raised-line drawings (Heller et al., 2003). The above advantages of blind over sighted individuals in the processing of two-dimensional haptic stimuli may be associated with experience in using tactile aids and with the practice of active exploratory strategies (cf. D'Angiulli \& 
Kennedy, 2001; Davidson, 1972; Perkins \& Gardiner, 2003; Russier, 1999; Symmons \& Richardson, 2000), as well as greater haptic sensitivity of blind than sighted participants (Sathian \& Prather, 2006). In view of the blind individuals' capacities related to the processing of shapes perceived haptically, we predict that: (H1) Congenitally blind participants learn 2D embossed right-angle-shapes faster than sighted individuals.

Blind participants are as successful as sighted individuals in tasks involving retention of a spatial haptic stimulus in working memory (cf. Cornoldi \& Vecchi, 2003; Vecchi, 1998; Vecchi, Monticell, \& Cornoldi, 1995). Research into recognition of two-dimensional shapes established that congenitally blind individuals recognize shapes equally accurately (Bailes \& Lambert, 1986; Picard et al., 2010), or indeed more accurately (Pathak \& Pring, 1989 - the study of children), in comparison to blindfolded sighted participants. Due to the haptic perception and recognition capacities in people who are blind, we predict that: (H2) Congenitally blind participants recognize 2D embossed right-angleshapes more accurately than sighted individuals.

Vecchi, Monticelli and Cornoldi (1995) conducted a series of experiments investigating variables affecting visuo-spatial working memory capacity. They claim that visuo-spatial working memory has two components, a passive store and active imagery operations. Their first experiment consisted of two stages and involved blind and sighted participants. The stimuli consisted of a $5 \times 5$ two-dimensional matrix of squares. During the first stage, participants performed two types of tasks in succession: 1) an active pathway task, which involved tracking a verbally presented pathway through a matrix; 2) a passive positions task, which involved memorizing the spatial position of squares presented in a matrix explored through touch. In the active pathway task, blind partici- pants were less successful than sighted participants in identifying the final position of the target when the task was more complex, i.e. the pathway involved more movements in the $2 \mathrm{D}$ matrix. In the passive positions task, the increasing complexity impaired the performance in both groups: memory for the spatial configuration was better when it comprised four targets rather than eight. During the second stage, participants had to perform the two tasks (active pathway and passive positions) simultaneously. In the active pathway task, differences in the performance of the blind and sighted participants can clearly be seen - blind participants performed this task significantly worse than sighted participants. This result is in line with other studies using tasks requiring active visuospatial working memory, which have shown that such tasks present a greater challenge to blind participants than sighted participants (cf. Cornoldi \& Vecchi, 2003; Vecchi, 1998). In the dual task condition, performance on the passive positions task was influenced by memory load in the sighted group only. When they had to remember five target objects the performance of the active pathway task was worse than when they only had to remember two, whereas the blind group performed similarly in both conditions. The results of this second stage suggest that blind people find retaining a spatial stimulus in working memory a relatively easy task, regardless of the complexity of the additional active task. In our study, the recognition task can be treated as a dual task situation - it requires retaining the representation of a model figure in memory (passive task) and creating a representation of a test figure explored by touch (active task - due to the sequentiality of haptic cognition, the creation of mental representation requires the assembling of elements into a whole). Despite the active nature of creating a mental representation of the haptic pattern, blind people cope with such tasks as well or even better than sighted people (cf. Ballesteros et 
al., 2005; Theurel et al., 2012). Therefore, we expect that: (H3) Only sighted participants more accurately recognize $2 \mathrm{D}$ tactile right-angleshapes with fewer angles, compared to a greater number of angles (we do not predict the same difference in participants with blindness).

The blind group's performance on the passive positions tasks (both alone and in the dual task condition) was somewhat inconsistent (Vecchi et al., 1995). When performing the task alone, blind participants did less well in the complex condition, whereas in the dual task stage their performance was similar in the simple and complex stimulus conditions. However, the number of target objects to be retained in memory in the simple and complex conditions differed in the single and dual task conditions (single task: 4 and 8; dual task: 2 and 5). This, together with the difference in experimental procedure, makes direct comparison problematic. It is possible that the memory load only affects the passive component of visuo-spatial working memory when the number of elements to be remembered exceeds Miller (1956) the "magic" number (7). This is the maximum number of elements that can be held in working memory efficiently.

While comparing the function of visuo-spatial working memory in congenitally blind and in sighted individuals, we should consider mental strategies employed by these two populations. In experiments known to us, where researchers investigated strategies used by blind and sighted participants to encode raised-line patterns and retain these in memory, i.e. in tasks engaging the passive component of visuo-spatial working memory, no relationships were identified between the applied strategy and accuracy of the pattern recognition (Lebaz, Picard, \& Jouffrais, 2010; Picard et al., 2010). These studies suggest that sighted participants tend to more often employ visual strategies, i.e. visuospatial strategy alone or in combination with verbal or kinesthetic strategy. On the other hand blind individuals (studies have focused on both early and late blind participants) prefer non-visual strategies, i.e. spatial, kinesthetic and verbal, each of these may be employed either as a leading strategy or in combination with one of the remaining strategies. Visuo-spatial strategy, which involves creating a mental representation of a pattern, requires visualization. Unlike visual strategy, spatial strategy lacks visual clues and mental representation focuses on the spatial arrangement, e.g. directions or relationships between components of the figure. Verbal strategy involves making a description of a spatial stimulus with the use of words (e.g., right, left, oblique, zigzagging). Kinesthetic strategy makes use of motor memory. We expect that similar differences in the applied strategies as reported by Lebaz and colleagues (2010), and Picard and associates (2010), will be identified in congenitally blind and sighted participants, in a study designed to use different research material $-2 \mathrm{D}$ tactile right-angle-shapes. The following hypothesis was formulated: (H4) While memorizing a figure, sighted individuals use visual strategies more often than congenitally blind participants. The study also investigated the following problem: (P1) What specific strategies, within the categories of visual and non-visual (spatial, verbal, kinesthetic or mixed) strategies are used by individuals with and without visual experience?

To the best of our knowledge there is no research directly comparing memorizing of nonfigurative 2D spatial stimuli presented against a grid and without a grid $^{1}$. In a single experiment, researchers displayed spatial haptic stimuli either against a grid (Cornoldi \& Vecchi, 2003; Vecchi, 1998; Vecchi et al., 1995), or without a grid, in an embossed frame (Bailes \& Lambert, 1986) or on a completely empty surface,

\footnotetext{
1 Although, such conditions were compared by Szubielska and Zabielska-Mendyk (2018), but in the experiment on mental rotation.
} 
on a Swell paper - in the latter case the edge of the paper constituted the frame (Picard et al., 2010). On the one hand, lines of the grid may be a distractor, making it difficult to distinguish the raised-line figure from the background. Heller and colleagues (2003) showed that in a task which involved tracing, with preferred index finger, of a figure embedded in a raised-line drawing, the accuracy decreased if the figure was presented against a background consisting of a greater number of intersecting lines, in comparison to the background consisting of fewer lines. The effect was observed in groups of both blindfolded sighted participants and congenitally blind individuals. On the other hand, a grid facilitates the use of verbal strategy of memorizing, called coordinate XY, which involves remembering coordinates of specific squares of the grid occupied by elements of the memorized stimulus (Szubielska, 2014; Vanlierde $\&$ Wanet-Defalque, 2004). Where the time allowed for exploring the shape is unlimited, individuals employing this strategy can accurately memorize the coordinates of all squares of the grid, which are occupied by the figure. In turn, sighted people prefer visualization strategy (Vanlierde \& Wanet-Defalque, 2004, although Szubielska, 2014 stated that they use it as frequently as the XY verbal strategy). To deter- mine whether a background in the form of a grid makes it easier or more difficult to memorize 2D right-angle-shapes by people who are blind or sighted, the following problem was investigated: (P2) Do people who are blind, solve the task most accurately when they both learn and recognize a figure in the context of the grid, due to their preferred imagery strategies, while the sighted people - when they learn and recognize a pattern in the context of the frame?

\section{Method}

\section{Participants}

The final sample consisted of 22 individuals (6 women), half of whom were congenitallyblind (they were at most able to sense light and had never been able to see) and half of whom had normal vision. There were initially 12 blind participants, but the scores of one blind female participant and her sighted match had to be discarded because the blind participant often confused the model and test figures; this may have reflected a misunderstanding of the instructions or an attention deficit. Sighted controls were matched to blind participants with respect to gender, age, handedness and level of education. The mean age of the blind group was 24.27

Table 1 Detailed information on the congenitally blind participants

\begin{tabular}{lllllll} 
& Sex & Age & Handedness & Education & Aetiology & $\begin{array}{l}\text { Light/darkness } \\
\text { sensitivity }\end{array}$ \\
\hline CB1 & M & 25 & Right & High & Retinoblastoma & No \\
CB2 & M & 30 & Right & High & Optic nerve atrophy & Yes \\
CB3 & M & 27 & Right & High & Retinopathy of prematurity & Yes (left eye) \\
CB4 & M & 24 & Right & Secondary & Retinopathy of prematurity & Yes \\
CB5 & F & 22 & Right & Secondary & Optic nerve hypoplasia & Yes \\
CB6 & M & 18 & Right & Secondary & Retinopathy of prematurity & No \\
CB7 & M & 36 & Right & Secondary & Retinopathy of prematurity & No \\
CB8 & M & 18 & Right & Secondary & Retinopathy of prematurity & Yes \\
CB9 & F & 23 & Right & Secondary & Optic nerve hypoplasia & Yes \\
CB10 & M & 25 & Left & Secondary & Retinoblastoma & No \\
CB11 & F & 19 & Right & Secondary & Optic nerve atrophy & No \\
\hline
\end{tabular}


years (range: 18 -36 years). All blind participants could read Braille and had some experience of using tactile graphics. The detailed characteristics of the groups are shown in Table 1. The mean age of the sighted group was 24.18 years (range: 18-36 years).

\section{Materials}

Testing material consisted of 80 tactile images, each with a surface area of 40 square centimeters; half were used in the learning stage (target figures) and the rest were presented in the recognition stage (test figures). Images consisted of an asymmetric, non-figurative planar shape, displayed either against a 100-square
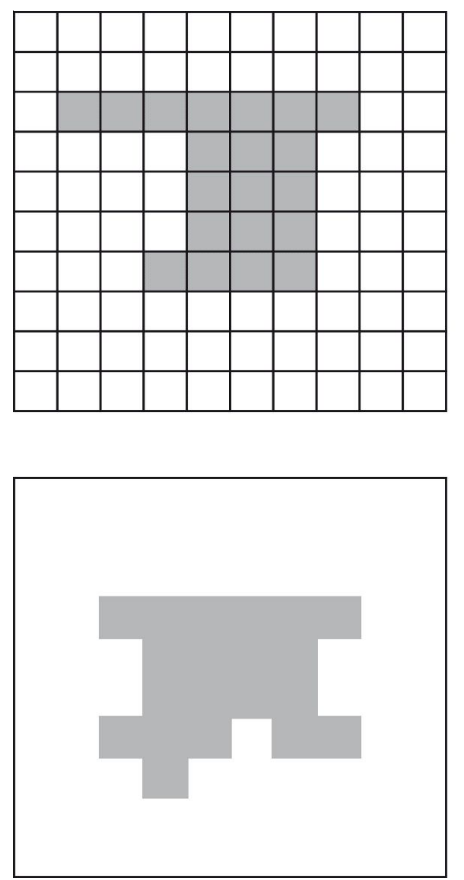

$\operatorname{grid}(2 \mathrm{~cm} \times 2 \mathrm{~cm})$ or in a frame (see Figure 1). In the frame condition the figure (convex; $0.5 \mathrm{~mm}$ high; dotted texture) was placed inside a $20 \mathrm{~cm}$ square with a raised profile ( $1 \mathrm{~mm}$ high). In the grid condition the texture of the raised grid ( $1 \mathrm{~mm}$ high) differed from that of the figure (convex; $0.5 \mathrm{~mm}$ high; dotted texture); we confirmed during a pilot study that the two textures were easy to distinguish. Each shape consisted of 20 square cells (the borders between cells were detectable when shapes were displayed against a grid; they were not perceptible when figures were presented in a frame). Target shapes varied in terms of number of angles, low: 10 angles or high: 20 angles (see Figure 1). All the angles were right angles.
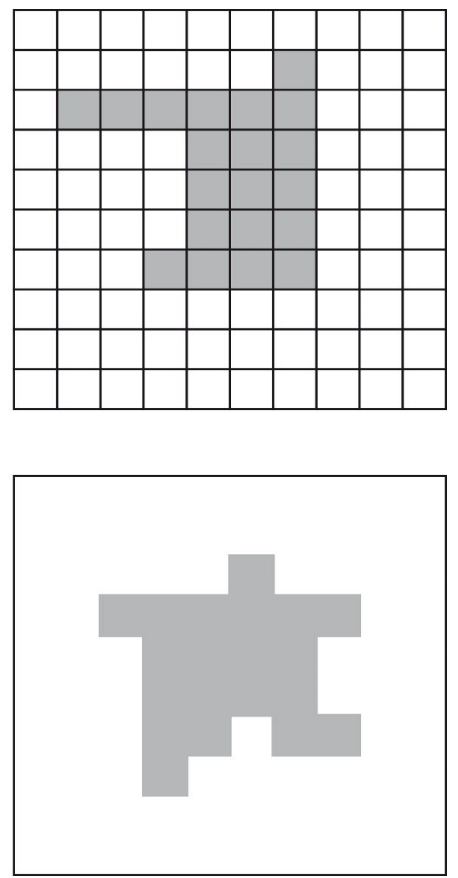

Figure 1 Examples of experimental stimuli. Model figures (left) and test figures (right) displayed against a grid and in a frame. Examples of figures with low-angle number are presented in the upper row, while figures with high-angle number are presented in the lower row. 


\section{Procedure}

The participants' task was to memorize a tactile target shape and there was no limit to the amount of time they could take. The instructions were as follows: "Your task is to memorize the shape of a figure, which you will explore by touch. Tell the experimenter when you think you have memorized the figure". Participants decided when they had memorized the figure; the learning phase ended when they made their declaration and the recognition test was administered without delay. This consisted of presenting a test figure and requiring the participant to decide if it was identical in shape to the target figure. Test figures were either identical to the target figure or differed with respect to the position of one cell (see Figure 1). Performance accuracy was assessed using a binary scale, with points scored for both hits and correct rejections. Sighted participants were blindfolded throughout the experiment.

Both target and test figures could be presented in the grid or in the frame, giving the following conditions: 1) learning with frame-recognition with frame(frame-frame), 2) learning with framerecognition with grid(frame-grid), 3) learning with grid-recognition with grid (grid-grid), 4) learning with grid - recognition with frame (gridframe). The pairings were presented in random order. Each pairing of contexts was used for ten trials (fiveeach for stimuli with low and high numbers of angles; also in random order). Before performing each condition participants performed three training trials which involved figures containing 12 to 18 angles. After completion of four conditions participants were asked to describe how they coded the 2D tactile shapes (see Cornoldi et al., 2009, and Picard et al., 2010 for a similar procedure).

A stopwatch was used to measure learning time. Learning time was measured from the moment the tactile graphic was first touched to the moment the participant informed the experimenter that he or she had memorized the model.

\section{Results}

\section{Exploration Time in the Learning Phase}

We performed ANOVA with mean exploration time as the dependent variable, number of angles (low; high), learning condition (frame; grid) and testing condition (frame; grid) as within-participants factors and visual status (sighted; congenitally blind) as between-participants factor. Descriptive statistics for mean learning time are presented in Table 2.

Mean exploration time was longer in sighted participants $(M=113.28, S E=13.40)$ than in blind participants $(M=46.24, S E=13.40 ; F(1,20)=$ $\left.12.51, M S E=15805.03, p=.002, \eta^{2}=.38\right)$.

It took longer to explore stimuli consisting of 20 angles $(M=92.22, S E=9.63)$ than stimuli consisting of 10 angles $(M=67.30, S E=10.26$; $F(1,20)=16.98, M S E=1630.01, p<.001, \eta^{2}=$ 46).

In the learning condition mean exploration time was longer when stimuli were presented against a grid $(M=95.46, S E=12.17)$ rather than in a frame $(M=64.06, S E=7.11 ; F(1,20)=$ $\left.25.98, M S E=1669.82, p<.001, \eta^{2}=.56\right)$. The same effect was observed for learning condition - mean exploration time was longer when the participant knew that a test figure will be presented against a grid $(M=88.07, S E=12.38)$ not in a frame $(M=71.44, S E=7.49 ; F(1,20)=$ 4.62, $\left.M S E=2632.05, p=.044, \eta^{2}=.19\right)$.

There were no significant interactions (double, triple, or fourfold) of visual status with the other factors analyzed ( $p s>.05)$.

\section{Performance Accuracy in the Recognition Phase}

We performed ANOVA with mean accuracy as the dependent variable and number of angles 
Table 2 Descriptive statistics for exploration time in the learning phase (in seconds). Table presents mean learning time (M), standard deviation (SD), minimum (Min) and maximum (Max) for every experimental condition for both sighted and congenitally blind participants

\begin{tabular}{llrrrr}
\hline Condition & & $M$ & $S D$ & Min & Max \\
\hline \multirow{2}{*}{ Frame-Frame Low-angle } & Blind & 19.75 & 10.61 & 5.65 & 36.93 \\
\cline { 2 - 6 } Frame-Grid Low-angle & Sighted & 66.75 & 38.19 & 16.58 & 135.00 \\
\hline \multirow{2}{*}{ Grid-Frame Low-angle } & Blind & 29.27 & 22.13 & 6.35 & 71.89 \\
\cline { 2 - 6 } & Sighted & 75.96 & 57.86 & 19.73 & 223.14 \\
\hline \multirow{2}{*}{ Grid-Grid Low-angle } & Blind & 39.23 & 23.86 & 8.26 & 81.44 \\
\cline { 2 - 6 } & Sighted & 107.84 & 43.23 & 41.45 & 198.93 \\
\hline \multirow{2}{*}{ Frame-Frame High-angle } & Blind & 46.72 & 30.12 & 9.42 & 116.05 \\
\cline { 2 - 6 } & Sighted & 126.95 & 55.85 & 45.86 & 211.37 \\
\hline \multirow{2}{*}{ Frame-Grid High-angle } & Blind & 40.42 & 24.86 & 7.45 & 82.88 \\
\cline { 2 - 6 } & Sighted & 106.73 & 67.61 & 26.03 & 233.43 \\
\hline \multirow{2}{*}{ Grid-Frame High-angle } & Blind & 67.25 & 73.10 & 5.77 & 216.60 \\
\cline { 2 - 6 } & Sighted & 132.25 & 115.04 & 28.17 & 441.28 \\
\hline \multirow{2}{*}{ Grid-Grid High-angle } & Blind & 62.31 & 40.56 & 7.88 & 136.82 \\
\cline { 2 - 6 } & Sighted & 128.52 & 49.60 & 43.48 & 222.56 \\
\hline & Blind & 64.93 & 41.11 & 9.01 & 146.29 \\
\cline { 2 - 6 } & Sighted & 161.24 & 83.45 & 45.20 & 310.93 \\
\hline
\end{tabular}

(low; high), learning condition (frame; grid) and testing condition (frame; grid) as within-subjects factors and visual status (sighted; congenitally blind) as the between-subjects factor. Descriptive statistics for accuracy are presented in Table 3.

There were no main effects of number of angles $(F(1,20)=.02, p=.882)$, learning condition $(F(1,20)=3.33, p=.083)$ or visual status $(F(1,20)=0.01, p=.933)$.

Mean accuracy was higher when test stimuli were presented in a frame $(M=3.81, S E=.12)$ rather than against a grid $(M=3.38, S E=.20$; $\left.F(1,20)=4.98, M S E=1.65, p=.037, \eta^{2}=.20\right)$. There was an interaction of number of angles and learning condition $F(1,20)=6.59, p=.02$. Bonferroni post-hoc test showed that when participants were learning stimuli presented in a frame, the accuracy was higher for low angle condition $(M=3.91, M S E=.16)$ than for high angle condition $(M=3.57, M S E=.16, p=.049)$. Also, for low angle condition the accuracy was higher when the participant learned the stimuli in a frame $(M=3.91, M S E=.16)$ than against a $\operatorname{grid}(M=3.25, M S E=.24, p=.012)$.

There was also a three-way interaction between learning condition, testing condition and visual status $(F(1,20)=6.80, M S E=1.24, p=$ $.017, \eta^{2}=.25$ ) (see Figure 2). Bonferroni posthoc test revealed that only in the sighted group there were differences in accuracy. When sighted participants were learning in a frame condition and were tested in a frame condition $(M=4.41, M S E=.17)$ the accuracy was higher than when they were learning in a frame condition and tested against a grid condition $(M=$ $3.14, M S E=.31 ; p<.001)$. The accuracy was also higher for sighted participants when they learned in a frame condition and tested in a frame condition $(M=4.41, M S E=.17)$ than when 
Table 3 Descriptive statistics for performance accuracy in the recognition phase. Table presents mean accuracy (M), standard deviation (SD), minimum (Min) and maximum (Max) for every experimental condition for both sighted and congenitally blind participants

\begin{tabular}{llcccc}
\hline Condition & & $M$ & $S D$ & Min & Max \\
\hline \multirow{2}{*}{ Frame-Frame Low-angle } & Blind & 4.27 & .65 & 3 & 5 \\
\cline { 2 - 5 } Frame-Grid Low-angle & Sighted & 4.45 & .93 & 2 & 5 \\
\hline \multirow{2}{*}{ Grid-Frame Low-angle } & Blind & 3.64 & .67 & 3 & 5 \\
\cline { 2 - 6 } & Sighted & 3.27 & 1.35 & 1 & 5 \\
\hline \multirow{2}{*}{ Grid-Grid Low-angle } & Blind & 3.45 & 1.37 & 1 & 5 \\
\cline { 2 - 6 } & Sighted & 3.09 & 1.45 & 0 & 5 \\
\hline \multirow{2}{*}{ Frame-Frame High-angle } & Blind & 3.18 & 1.54 & 1 & 5 \\
\cline { 2 - 6 } & Sighted & 3.27 & 1.56 & 0 & 5 \\
\hline \multirow{2}{*}{ Frame-Grid High-angle } & Blind & 3.55 & .93 & 2 & 5 \\
\cline { 2 - 6 } & Sighted & 4.36 & .67 & 3 & 5 \\
\hline \multirow{2}{*}{ Grid-Frame High-angle } & Blind & 3.36 & 1.36 & 1 & 5 \\
\cline { 2 - 6 } & Sighted & 3.00 & 1.26 & 1 & 5 \\
\hline \multirow{2}{*}{ Grid-Grid High-angle } & Blind & 3.64 & 1.03 & 2 & 5 \\
\cline { 2 - 6 } & Sighted & 3.64 & 1.12 & 2 & 5 \\
\cline { 2 - 5 } & Blind & 3.55 & 1.29 & 1 & 5 \\
\hline & Sighted & 3.73 & .90 & 2 & 5 \\
\hline
\end{tabular}
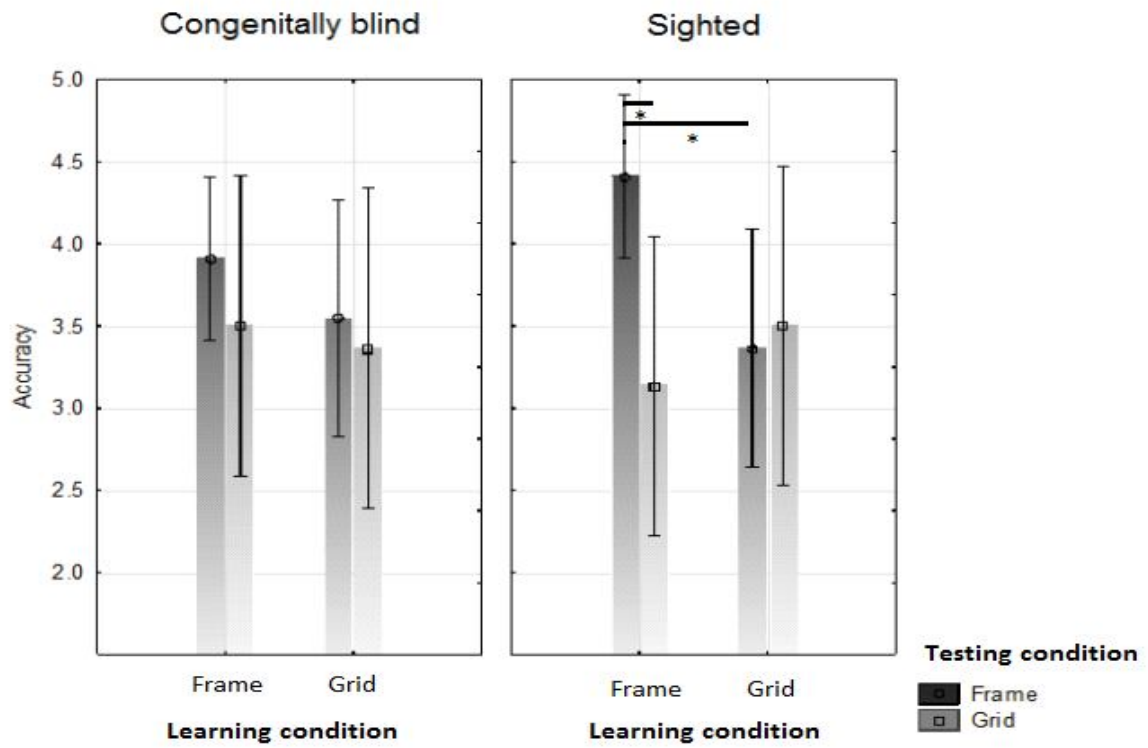

Figure 2 Interaction of accuracy (tactile pattern recognition performance) relative to learning condition, testing condition and visual status (error bars represent $+/$ - standard errors). 
they learned against a grid condition and tested in a frame condition $(M=3.36, M S E=.25 ; p=$ $.003 ; p<.001)$ (See Figure 2).

There were no more significant interactions (double, triple, or fourfold) of visual status with the other factors analyzed $(p s>.05)$.

\section{Self-Reported Strategies}

Each participant's verbal report was examined by two independent judges. They classified the reports according to the strategies used to memorize the tactile figures. The judges were provided with definitions and sample statements reflecting the use of visuo-spatial, spatial, verbal and kinesthetic strategy; these originated from the study by Picard and colleagues (2010). The statements of all the participants were assessed four times, concerning the four defined imagery strategies. The following strategy descriptions were given to the judges: 1) "Participants imagined how parts of the configuration or the whole pattern appeared, relying on visual imagery" - visuo-spatial strategy; 2) "Participants used descriptive words to encode spatial information" - verbal strategy; 3) "Participants imagined how parts of the configuration or the whole pattern appeared without recourse on visual cues - spatial strategy; 4) "Participants imagined how parts of the configuration or the whole pattern appeared relying on motor or kinesthetic information - kinesthetic strategy (Picard et al., 2010, p. 230). Sample statements by which it was identified that the participant used the 1) visuo-spatial, 2) verbal, 3) spatial, and 4) kinesthetic strategy were: 1) "I searched for outlines and drew a figure in my head", "I try to imagine the shape, see it with my mind's eye"; 2) "I counted cells, I counted the distance from the edge and the gap between elements", "I studied the figure from top to bottom, counting how many grates are in a row, how many empty grids were there"; 3 ) "First, I memorized the middle, then the periphery and associated the image with something known", "I memorized using the principle of a city plan (narrow fragments are streets, wider are squares); I memorize each piece, fragments; the whole shape does not mean anything to me"; 4) "I memorized the entire shape by running my finger along the edge", "I have a measuring tape in my fingers (width of the fingers, spacing) I am applying distance measure, checking on the sides or diagonally". The judges agreed on 80 out of 88 cases. The disparities in their decisions were resolved during a discussion.

Sighted participants used the visuo-spatial strategy significantly more frequently than the blind participants, $\chi^{2}(1)=22, p<.001$, and sighted participants used the spatial strategy significantly less frequently than the blind participants, $\chi^{2}(1)=22, p<.001$. All the sighted participants applied visual strategies and none of them used spatial strategies, and all the blind participants employed non-visual strategies and none of them declared using visual strategies. The groups of blind and sighted participants did not differ in the frequency of using verbal strategy, $\chi^{2}(1)=.75, p=.386$, or kinesthetic strategy, $\chi^{2}(1)=.31, p=.58$.

It was determined that seven different strategies were used (see Table 4), including four types classified as visual, and three types classified as non-visual strategies. Using the Q Cochran's test, the frequency of application of those strategies in the groups of blind and sighted people was examined. There were no significant differences in the group of people with blindness, $Q(2)=5.09, p=.078$, or sighted, $Q(3)=3.18, p=.364$.

In their statements the participants spontaneously made comments regarding whether the grid facilitated or distracted memorization of a figure placed against it. Five congenitally blind and five sighted participants admitted directly that grids interfered with their performance. Four blind participants (and no sighted individuals) admitted directly that the grids were 
Table 4 Numerical distributions of the congenitally blind and blindfolded sighted participants according to the strategy used

\begin{tabular}{lcc}
\hline Strategy & Blind (N) & Sighted (N) \\
\hline Non-visual & 11 & 0 \\
spatial & 3 & 0 \\
spatial and verbal & 7 & 0 \\
spatial, verbal and kinesthetic & 1 & 0 \\
\hline Visual & 0 & 11 \\
visuo-spatial & 0 & 5 \\
visuo-spatial and verbal & 0 & 3 \\
visuo-spatial and kinesthetic & 0 & 1 \\
visuo-spatial, verbal, and kinesthetic & 0 & 2 \\
\hline
\end{tabular}

helpful. Furthermore, eight blind participants and five sighted participants reported that they counted the squares while memorizing the figures.

\section{Discussion}

The study tested four research hypotheses related to memorization of $2 \mathrm{D}$ embossed rightangle-shapes: (H1) Congenitally blind participants learn shapes faster than sighted individuals; (H2) Congenitally blind participants recognize 2D embossed right-angle-shapes more accurately than sighted individuals; (H3) Only sighted participants will more accurately memorize shapes with fewer angles compared to a greater number of angles; (H4) While memorizing a figure, sighted individuals use visual strategy more often than people who are congenitally blind. The study also investigated two questions: (P1) What specific strategies - spatial, verbal, kinesthetic or mixed, are used by blind and sighted individuals? (P2) Do people who are blind, solve the task most accurately when they both learn and recognize a figure in the context of the grid, due to their preferred imagery strategies, while the sighted people when they learn and recognize a pattern in the context of the frame?
The findings support the first hypothesis. The congenitally blind participants needed less time than the sighted individuals to learn 2D embossed right-angle-shapes, regardless of the number of angles in the figures and regardless of the form of presentation, against a grid or in a frame. The evidence is consistent with earlier studies suggesting that the time required for exploring a stimulus by touch is longer in the case of sighted individuals, compared to blind individuals (Heller, 2006; Postma et al., 2007).

The second hypothesis was not confirmed. Participants with congenital blindness and blindfolded sighted participants did not significantly differ in the accuracy of recognizing 2D embossed right-angle-shapes. Finding no significant differences between people who are blind and those who see but are blindfolded in recognizing tactile patterns is not an isolated result (cf. Bailes \& Lambert, 1986; Cornoldi \& Vecchi, 2003; Picard et al., 2010; Vecchi, 1998; Vecchi, Monticell, \& Cornoldi, 1995). It is possible that visual experience does not differentiate the performance of tasks that require tactile perception. Differences obtained in research in this area are rare (e.g., Pathak \& Pring, 1989), and studies with statistically significant results are preferred in the scientific literature (cf. Picard et al., 2010). 
The third hypothesis was not confirmed either. The accuracy of recognizing figures with fewer or larger number of angles did not differ significantly, neither in the blind and nor in the sighted participants. Increasing complexity did not impair the performance in either group, which is inconsistent with the other studies' results (Vecchi et al., 1995). The fact that we did not limit exploration time may explain the discrepancies between our findings and those of Vecchi and colleagues (1995) (importantly, these researchers applied spatial stimuli which differed from ours, and this may have contributed to the different findings acquired in the two experiments). In our study the participants needed more time to learn the figures with more angles, compared to the figures with fewer angles, which means that the effect of complexity of $2 \mathrm{D}$ tactile right-angle-shapes in the load of the passive component of visuo-spatial working memory was observed in the dependent variable of learning time but not in recognition accuracy.

The fourth hypothesis and the first research problem was related to the strategies of memorizing embossed figures applied by the congenitally blind and sighted participants. The hypothesis was verified positively. It was determined that the blindfolded sighted individuals employed visual strategies only, while the blind participants employed non-visual strategies ${ }^{2}$. These findings are consistent with those reported by Picard and colleagues (2010), and Vanlierde and Wanet-Defalque (2004). It was confirmed that in the process of creating mental representation of a pattern, sighted participants try to visualize it while blind individuals with no visual experience refer to spatial cat-

\footnotetext{
2 The result of people who are congenitally blind may seem obvious, but there are some controversial reports that individuals who are blind can use visual imagery (Bertolo et al., 2003), and that early blind participants can use the visualization strategy (Vanlierde \& Wanet-Defalque, 2004).
}

egories. Like Picard and colleagues (2010), we established that strategies in both the visual and non-visual categories include pure (visuospatial and spatial, respectively) and mixed strategies. In the visual category the following mixed strategies were identified: visuo-spatial combined with verbal, visuo-spatial combined with kinesthetic, as well as visuo-spatial combined with both verbal and kinesthetic. The frequency of application of those strategies did not differ significantly in the group of sighted individuals. Mixed strategies in the non-visual category included spatial strategy combined with verbal, as well as spatial combined with verbal and kinesthetic. The frequency of application of those strategies did not differ significantly in the group of people with blindness.

The second research question was related to the effect of the grid and the frame in the performance of a memory task by people who are blind or sighted. Regardless of visual status, the grid condition made the task of learning embossed figure more complex - as evidenced by the interaction effect of the number of angles and learning condition for accuracy (when participants were learning stimuli presented in a frame, the recognition accuracy was higher for low angle condition than for high angle condition; for low angle condition the recognition accuracy was higher when participant learn stimuli in a frame than against a grid). But, in the case of the sighted participants, a grid was a more considerable distractor than in case of individuals with congenital blindness. In the group of blindfolded sighted participants, the accuracy was higher, when the shape: was learned in a frame and tested in a frame than learned in a frame and tested against a grid; was learned in a frame and tested in a frame than learned against a grid and tested in a frame. In the group of people who are blind, there were no differences in the accuracy of figure recognition, which were learned and which were recognized in different conditions (frame or grid). 
During conversations after the end of the experiment nearly half of the participants (both blind and sighted) spontaneously reported that the grid interfered with the performance of the task. None of the sighted participants found the grid to be helpful. Some participants with blindness admitted that the grid was of help to them, but it may have been related to the used memory strategy. Eight of the blind participants (nearly $73 \%$ of the group) reported that in order to memorize the figure they counted the squares, which means they used a strategy similar to coordinate XY strategy (cf. Szubielska, 2014; Vanlierde \& Wanet-Defalque, 2004). Interestingly, even when the grid was not there, some participants tried to employ this strategy, as illustrated by a statement of one of the participants with blindness, "I tried to superimpose a grid over the figure". The fact that the grid is a distractor in exploring a pattern placed against it is also reflected by the longer learning time of target figures when they were placed against a grid than when they were placed in a frame. Interestingly, participants also tended to spend more time learning target figures when they expected the test figure to be presented against a grid (frame-grid; grid-grid) than when they expected it to be placed in a frame (frame-frame; grid-frame). In the interpretation of the results we will again refer to the time-consuming verbal XY strategy (cf. Szubielska, 2014; Vanlierde \& Wanet-Defalque, 2004), possibly employed by the participants (both blind and sighted people), who reported having counted the squares. As a reminder, prior to each series of tasks, the participants performed test trials, therefore they always knew beforehand the condition of the target and the test figures presentation. Keeping in mind the comment contributed by one of the participants, who said that he tried to superimpose the grid onto the figure, we can suspect that when they expected the test figure to be displayed against a grid, even if at the exploration stage the figure was presented in a frame, some participants tried to count which squares of the imagined grid were occupied by the figure.

Distraction effects of the complex grid background in the accuracy of the sighted participants' performance may have been linked to the difficulty in distinguishing the figure from the background (Heller et al., 2003) or with the difficulty of visualizing it in the context of a grid (all sighted participants used visual memory strategies). Increasing complexity of an imagined stimulus results in the creation of more degraded visual image (Kosslyn, 1975).

The present study supports the claim made by Vecchi and colleagues (Cornoldi \& Vecchi, 2003; Vecchi, 1998) that individuals without visual experience do not have an impairment in the passive component of visuo-spatial working memory compared with sighted individuals. The findings are consistent with results of experiments which have shown that congenitally blind participants memorize two-dimensional shapes at least as accurately as sighted individuals (Bailes, Lambert, 1986; Pathak \& Pring, 1989; Picard et al., 2010; Vecchi, Monticelli, \& Cornoldi, 1995). They also reflect significant ability of blind individuals to create accurate representations of $2 \mathrm{D}$ non-figurative spatial stimuli and maintain it in working memory, which previously has been demonstrated in perception tasks (Alary et al., 2008; Theurel et al., 2012; Ballesteros et al., 2005).

Limitations of the study are related to the number of participants. Small groups of participants were examined, due to the fact that the population of people who are blind from birth, without additional disabilities, is limited (moreover, some of these people have repeatedly been asked to participate in psychological research and are reluctant to take part in the next). Quite often congenital blindness is accompanied by intellectual disability - this situation excludes the participation of a person from an experiment such as ours. 


\section{References}

Alary, F., Goldstein, R., Duquette, M., Chapman, C. E., Voss, P., \& Lepore, F. (2008). Tactile acuity in the blind: A psychophysical study using a two-dimensional angle discrimination task. Experimental Brain Research, 187, 587-594.

Bailes, S. M., \& Lambert, R. M. (1986). Cognitive aspects of haptic form recognition by blind and sighted subjects. British Journal of Psychology, 77, 451-458.

Ballesteros, S., Bardisa, D., Millar, S., \& Reales, J. M. (2005). The haptic test battery: A new instrument to test tactual abilities in blind and visually impaired and sighted children. British Journal of Visual Impairment, 23, 11-24.

Bertolo, H., Paiva, T., Pessoa, L., Mestre, T., Marques, R., \& Santos, R. (2003). Visual dream content, graphical representation and EEG alpha activity in congenitally blind subjects. Cognitive Brain Research, 15, 277-284.

Cornoldi, C., Tinti, C., Mammarella, I. C., Re, A. M., \& Varotto, D. (2009). Memory for an imagined pathway and strategy effects in sighted and in totally congenitally blind individuals. Acta Psychologica, 130, 11-16.

Cornoldi, C., \& Vecchi, T. (2003). Visuo-spatial working memory and individual differences. Hove, New York: Taylor and Francis/Psychology Press.

D’Angiulli, A., \& Kennedy, J. M. (2001). Children's tactual exploration and copying without vision. International Journal of Rehabilitation Research, 24, 233-234.

Davidson, P. W. (1972). Haptic judgments of curvature by blind and sighted humans. Journal of Experimental Psychology, 93, 43-55.

Heller, M. A. (2006). Picture perception and spatial cognition in visually impaired people. In M. A. Heller, \& S. Ballesteros (Eds.), Touch and blindness. Psychology and Neuroscience (pp. 49-71). Mahwah, New Jersey: Lawrence Erlbaum Associates.

Heller, M. A., Wilson, K., Steffen, H., Yoneyama, K., \& Brackett, D. D. (2003). Superior haptic perceptual selectivity in late-blind and very-low-vision subjects. Perception, 32, 499-511.

Kosslyn, S. M. (1975). Information representation in visual images. Cognitive Psychology, 7, 341370 .

Lebaz, S., Picard, D., \& Jouffrais, C. (2010). Haptic recognition of non-figurative tactile pictures in the blind: Does life-time proportion without visual experience matter? Volume of the series Lecture Notes in Computer Science, 6192, 412-417.
Miller, G. A. (1956). The magical number seven, plus or minus two: Some limits on our capacity for processing information. Psychological Review, 63, 8197.

Pathak, K., \& Pring, L. (1989). Tactual picture recognition in congenitally blind and sighted children. Applied Cognitive Psychology, 3, 337-350.

Perkins, C., \& Gardiner, A. (2003). Real world map reading strategies. The Cartographic Journal, 40, 265-268.

Picard, D., Lebaz, S., Jouffrais, C., \& Monnier, C. (2010). Haptic recognition of two-dimensional raised-line patterns by early-blind, late-blind, and blindfolded sighted adults. Perception, 39, 224-235.

Postma, A., Zuidhoek, S., Noordzij, M. L., \& Kappers, A. M. (2007). Differences between early-blind, lateblind, and blindfolded-sighted people in haptic spatial-configuration learning and resulting memory traces. Perception, 36, 1253-1265.

Russier, S. (1999). Haptic discrimination of two-dimensional raised-line shapes by blind and sighted adults. Journal of Visual Impairment \& Blindness, 93, 421-426.

Sathian, K., \& Prather, S. C. (2006). Cerebral cortical processing of tactile form: Evidence from functional neuroimaging. In M. Heller, \& S. Ballesteros (Eds.), Touch and blindness: Psychology and neuroscience (pp. 139-155). Lawrence Erlbaum Associates, New Jersey.

Symmons, M., \& Richardson, B. (2000). Raised-line drawings are spontaneously explored with a single finger. Perception, 26, 621-626.

Szubielska, M. (2014). Strategies for constructing spatial representations used by blind and sighted subjects. Studia Psychologica, 56, 273-285.

Szubielska, M., \& Zabielska-Mendyk, E. (2018, in press). Mental rotation of figures explored by touch. A study on congenitally blind and sighted individuals. Roczniki Psychologiczne // Annals of Psychology, 21.

Theurel, A., Frileux, S., Hatwell, Y., \& Gentaz, E., (2012). The haptic recognition of geometrical shapes in congenitally blind and blindfolded adolescents: Is there a haptic prototype effect? PloS One, 7:e40251. doi: 10.1371 /journal.pone.0040251.

Vanlierde, A., \& Wanet-Defalque, M.-C. (2004). Abilities and strategies of blind and sighted subjects in visuo-spatial imagery. Acta Psychologica, 116, 205222.

Vecchi, T. (1998). Visuo-spatial limitations in congenitally totally blind people. Memory, 6, 91-102.

Vecchi, T., Monticellai, M. L., \& Cornoldi, C. (1995). Visuo-spatial working memory: Structures and variables affecting a capacity measure. Neuropsychologia, 33, 1549-1564. 